\title{
Characterisation of Gunshot Residue Particles using Self-Consistent Ion Beam Analysis
}

\author{
MWebb, C Jeynes \\ University of Surrey Ion Beam Centre, Guildford, GU2 7XH, England
}

Published as: M.J.Bailey \& C.Jeynes, Nucl. Instrum. \& Methods B 267(12-13):2265226815 Jun 2009; http://dx.doi.org/10.1016/j.nimb.2009.03.031

\begin{abstract}
Samples of gunshot residue were collected from the hands of different shooters who had fired guns containing different respective ammunition powders. Individual particles within the samples were studied with particle-induced X-ray emission and backscattering spectroscopy using a $2.5 \mathrm{MeV} \mathrm{H}^{+}$beam focused to $\sim 4 \mu \mathrm{m}$ and selfconsistent fitting of the data. The compositions of the residue particles were determined unambiguously and with a higher sensitivity to trace elements than conventional methods.
\end{abstract}

Keywords: PIXE, RBS, EBS, Gunshot Residue, Simulated Annealing

\section{Introduction}

Identification of gunshot residues found on suspects has challenged forensic scientists for many years [1]. Energy dispersive spectroscopy (EDS) on the scanning electron microscope (SEM) is used routinely by police authorities to identify gunshot residue, and it has been shown that SEM-EDS can be used to distinguish between some residues from different types of ammunition powders [2]. We shall show that particle-induced X-ray emission (PIXE) with a microbeam is a more powerful technique to characterise such materials because of the much higher sensitivity to trace elements.

PIXE can be quantified absolutely without the use of standards, provided that the elemental composition of the material on the exit path of the X-rays is known, which is the case if the backscattered particle signal is collected and correctly analysed. However, until recently it has not been possible to analyse the photon and particle spectra self-consistently, that is, in an integrated code that uses the elemental specificity of the PIXE to overcome the poor mass resolution of the particle spectra, together with the compositional depth profile sensitivity of the particle spectra to correctly calculate the X-ray absorption on the exit path. In fact, the main PIXE codes could not correctly calculate absorption where the same element was in multiple layers.

We have incorporated the DATTPIXE code [3] into an open source C module LibCPIXE and integrated it [4], into DataFurnace [5], which is a fitting code using simulated annealing as a global minimisation algorithm as well as the local minimisation algorithms commonly used. DataFurnace has powerful facilities for handling multiple spectra simultaneously. The new PIXE/backscattering integrated code has been used to identify the corrosion product on a historic heliograph from 1827 [6], to quantify the pigment used on a $19^{\text {th }}$ century reproduction of Frans Hals' 
La Bohémienne (1630) [7], and to determine the $\mathrm{O}$ content of nitric acid treated carbon nanotubes [8]. It has also been used to determine the position and composition of micro-inclusions in unexpected carbonaceous inclusions in impact glasses from the meteoritic impact crater near Mt.Darwin, Tasmania [9]

When a firearm is discharged, microscopic particles of gunshot residue (GSR) are deposited on the hands, clothing, skin and hair of the shooter. These GSR particles are condensation products of the high pressure, high temperature reactions that take place when a gun is fired, and are made up of material from various components of the gun including the primer, cartridge casing and the projectile. The particles range in size from a few nm to tens of microns in diameter and usually contain $\mathrm{Pb}, \mathrm{Ba}$ and $\mathrm{Sb}$.

Gunshot residues are frequently used in police casework to prove that a suspect has fired a gun, because the residues can remain on the clothing of the shooter for several days after the incident. However, suspects are often arrested by police officers who themselves may be contaminated with gunshot residue particles either from previous case work or from their own firearms. This leads to uncertainty over the origin of gunshot residue found on a suspect and a risk of assuming a false positive. This problem has come into the spotlight in the UK in the recent high profile case of Barry George, who was convicted of the murder of the television presenter Jill Dando, in which one particle of gunshot residue was found on his clothing. Defence lawyers have appealed by arguing that the gunshot residue was most likely to be contamination either from the arresting officers or from the analysis procedure. This has highlighted the need to be able to discriminate one type of gunshot residue over another.

Police authorities routinely use SEM-EDS to characterise gunshot residues. Forensic service providers in the UK have developed a classification scheme for gunshot residues, putting them into 5 different categories in accordance with their elemental composition [10]. It follows that if gunshot residue found on a suspect falls in to the same category as gunshot residue generated by a firearms officer, the evidence is inconclusive. Therefore there is a clear need to further subclassify gunshot residues.

We have recently demonstrated [11] that microbeam PIXE is capable of detecting trace elements in gunshot residues that cannot be detected by SEM-EDS. We have shown that some residues, which fall into the same broad category of GSR in SEMEDS terms, can be further sub-categorized using PIXE. It was concluded that quantitative treatment of the PIXE data could be useful to strengthen the discriminating power of the technique. A distinct advantage of PIXE over SEM-EDS is of course the ability to determine the major, minor and trace element concentrations in absolute terms.

\section{Materials and Methods}

Gunshot residue samples were collected from shooters at Bisley Rifle Range, Surrey, England. Residues were collected from four different firearms: a pistol, 2 rifles and a shotgun. The pistol residue was obtained by swabbing the shooters' hands with an SEM stub. The other residues were collected by shaking the spent cartridge cases onto respective SEM stubs. Both methods have been used in previously published work for the collection of gunshot residue [12]. A Cu particle finder grid was placed on top of the stubs. 
The particles were located using a Philips XL30 scanning electron microscope, fitted with an EDAX EDX system. The beam energy was $30 \mathrm{keV}$ and the detector was a $133 \mathrm{eV} \mathrm{Si}(\mathrm{Li})$ UTW (ultra-thin window) detector with a take-off angle of $45^{\circ}$. Individual GSR particles were located and imaged using BSE image.

After the SEM-EDS analysis, the same particles were located and analysed using a $2.57 \mathrm{MeV}$ proton beam, focussed to $\sim 4$ microns [13]. PIXE spectra were obtained using a $146 \mathrm{eV} \mathrm{Si}(\mathrm{Li})$ detector at a take-off angle of $45^{\circ}$ with a $130 \mu \mathrm{m} \mathrm{Be}$ filter to exclude protons from the detector. Backscattered particle spectra were simultaneously collected at a scattering angle of $155^{\circ}$ and a solid angle of 50msr. The backscattered particle image was used to locate the particles, together with the PbL X ray map. Point spectra were taken for each of the particles.

\section{Results}

The RBS spectra (taken from a point at the centre of each of the samples) were found to vary considerably. This variance can be understood by considering the effect of the particle size on the spectrum. There are three different cases which give rise to different RBS spectra, depending on whether the beam is (a) larger (b) equivalent to, or (c) smaller in size compared with the sample, as shown in Figure 1.

In Figure 1b, we consider an RBS spectrum from a particle of similar size to the beam. There is a high energy signal coming from the particle ( $\mathrm{Pb}: \mathrm{Ba}: \mathrm{Sb})$ and a low energy signal which comes from the carbon substrate on which the particle sits. The atomic composition of the carbon substrate was determined from a point analysis to be $\mathrm{C} 8 \mathrm{O} 1 \mathrm{~Pb} 0.001$. It is interesting to note that there is a tailing of the low energy edge of the signal from the particle and a corresponding slope on the signal from the buried carbon substrate. This is because closer to the edges of the particle, the beam sees a thinner layer of particle above the substrate than it does at the centre of the particle.

Using a simulation code (such as OMDAQ [14], routinely used for PIXE/RBS), it is difficult, if not impossible, to fit the backscattered particle spectrum because the ratio of particle: substrate that the beam "sees" varies with depth in accordance with the sample topography. However, using Datafurnace, which is routinely used to extract accurate depth profiles from particle spectra, the spectra can be accurately fitted, as shown in Figure 2. The PIXE data from this sample was fitted self consistently with the particle spectrum, and the fit is shown in Figure $2 b$. The data was fitted by allowing the substrate $(\mathrm{C} 8 \mathrm{O} 1)$ and the particle (modeled as a single molecule of $\mathrm{Pb}$ $76 \mathrm{Al} ?=5 \mathrm{Si} ?=4 \mathrm{Fe} ?=0.14 \mathrm{Cu} ?=2.33 \mathrm{Zn} ?=0.21 \mathrm{Sb} ?=4.7 \mathrm{Ba} ?=0.8$, which was allowed to take various compositions) to vary as a function of depth. The resulting depth profile is shown in Figure 2c, and the depth scale is consistent with the particle being slightly larger than the beam spot size of $4 \mu \mathrm{m}$.

In Figure 1a, we consider the case where the particle is smaller than the beam spot size. The particle that gave rise to the spectrum in Figure 1a was approximately $3 \mu \mathrm{m}$ in diameter, while the beam spot size was $\sim 4 \mathrm{x} 4 \mu \mathrm{m}$. The spectrum shows a decrease in the signal coming from the particle and an enhancement in the carbon signal coming from the substrate, as would be expected for a small (i.e. thinner) particle. There is also a strong surface $C$ signal (channel 162), coming from the area in which the beam is incident only on the substrate. Figure 3 shows the fitted PIXE and RBS data for 
this sample and the corresponding depth profile. The depth profile shows that the particle is smaller (thinner) than the particle in Figure $1 \mathrm{~b}$.

In Figure 1c, we consider the case where the particle is larger than the beam spot size. This particle is $\sim 50 \mu \mathrm{m}$ in diameter, and the spectrum comes from a spot on the centre of the particle. In this case, the substrate cannot be seen in the RBS spectrum because the sample is thick. In this analysis, the $\mathrm{Pb}$ was allowed to vary independently from the $\mathrm{Ba}$ and $\mathrm{Sb}$ signals with depth. This was necessary to fit the data, in contrast to the other samples. The resulting depth profile is shown in Figure 4c. However, the PIXE maps do not show any non-uniformity in the $\mathrm{Pb} \mathrm{L}$ and $\mathrm{Sb} \mathrm{K}$ signals. Additionally, it is known that $\mathrm{Pb}$, having a significantly lower melting point than $\mathrm{Sb}$ and $\mathrm{Ba}$, will often solidify as an outer shell in GSR particles [15]. The derived depth profile, in which the $\mathrm{Pb}$ signal increases with depth, is not consistent with this expected behaviour.

We propose that the spectrum of this particle is a strong function of its topography. For spherical particles, the surface presented to the beam is not flat, even when the particle is larger in diameter than the beam. This means that the calculated exit pathlength of both particles and photons is progressively overestimated as the interaction point is deeper in the sample. This has the effect that the low energy scattering is underestimated. The fitting software compensates for this by putting high mass particles deep in the sample, and distorting both the detector solid angles and the stoichiometry of the sample.

\section{Conclusions}

We have shown that spherical particles of gunshot residue can be successfully fitted by simultaneous treatment of the PIXE and backscattering data. The fits are excellent for all sizes of particle, but for large particles the results are incredible. The extracted depth profiles in this case are clearly distorted by the spherical geometry, and must also distort the PIXE results. We conclude that compositions obtained in this way by PIXE will be distorted by this topography effect also for the small particles, although to a lesser extent, since the absorption is relatively small for small particles.

Accurate work must take account of particle shapes; but for this application, differences between particles that depend on such relatively small corrections cannot be used to reliably discriminate between GSR particles 

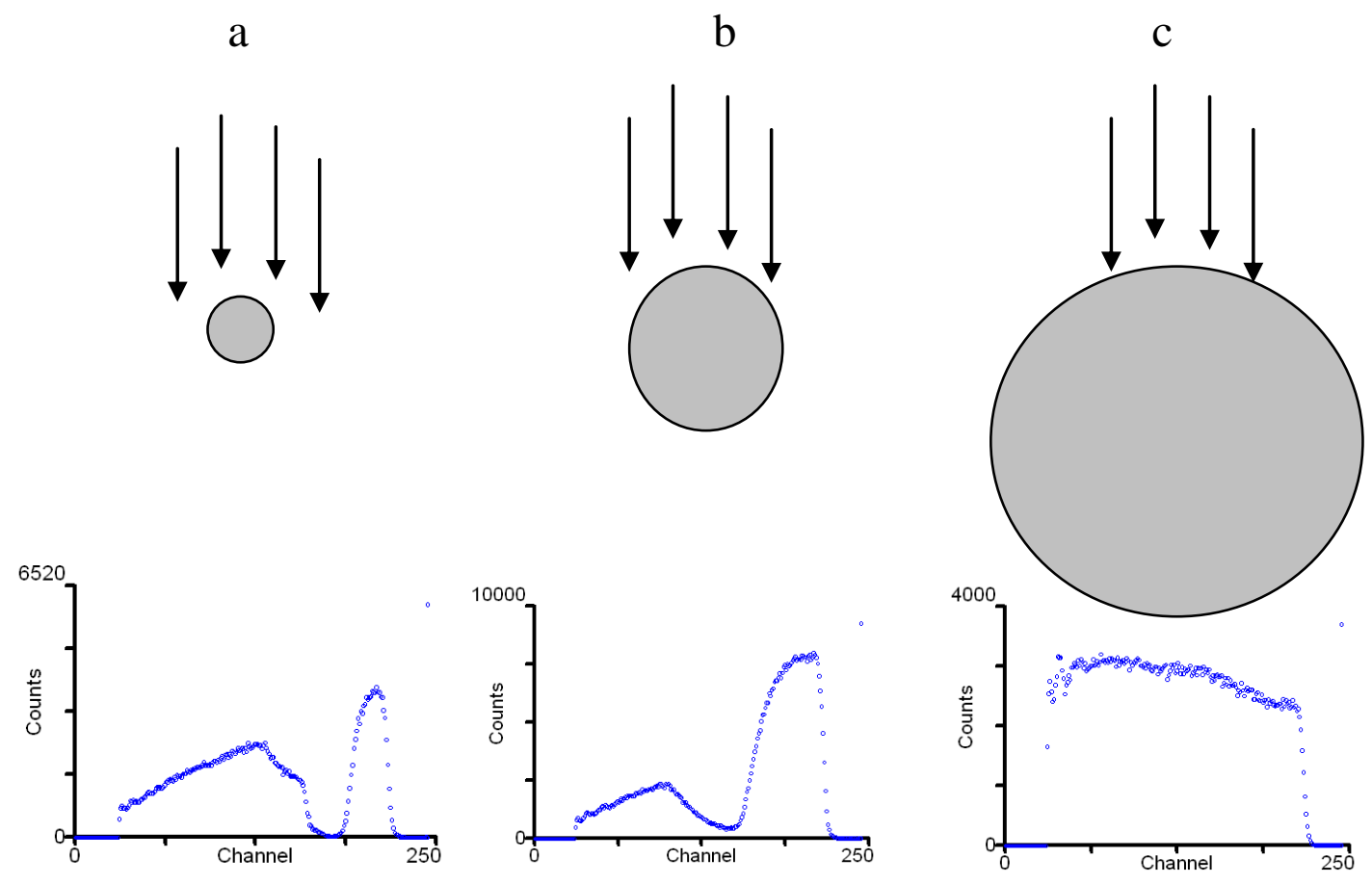

Figure 1 : typical RBS spectra from a particle that is (a) smaller (b) equivalent to and (c) larger than the analysing beam

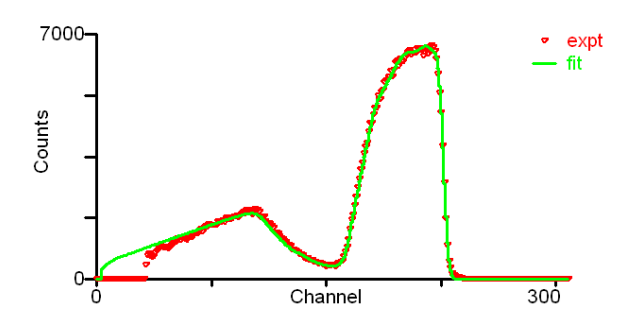

(a)

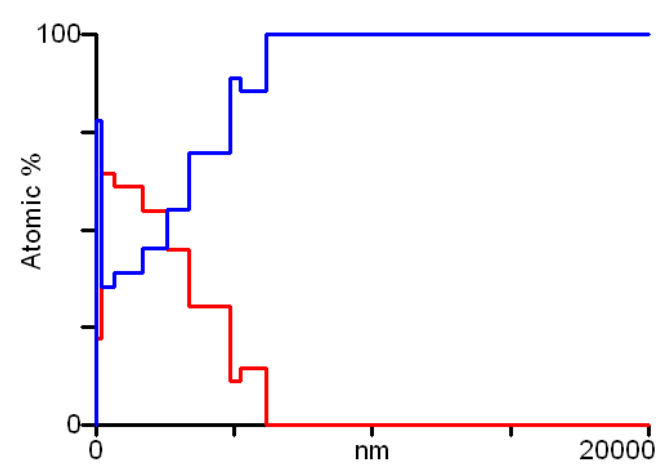

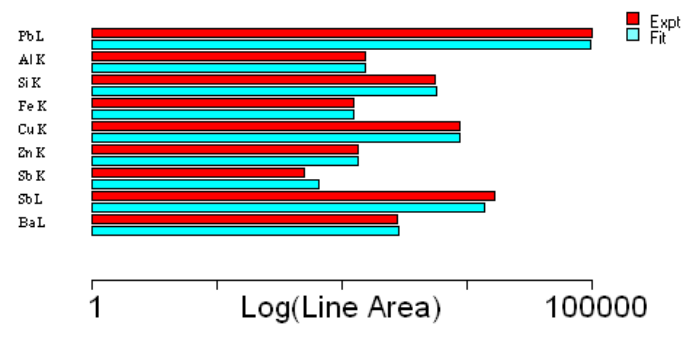

(b)

$\mathrm{Pb} 76 \mathrm{Al} ?=5$

C $801 \mathrm{~Pb}$

(c)

Figure 2 : (a) fitted RBS spectrum (b) simultaneously fitted PIXE spectrum and (c) corresponding depth profile for a particle of a similar diameter to the beam 


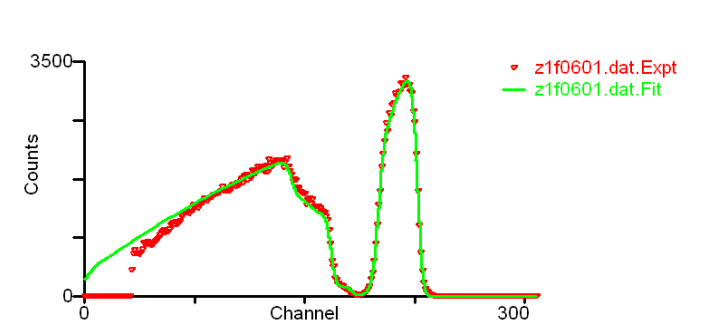

(a)

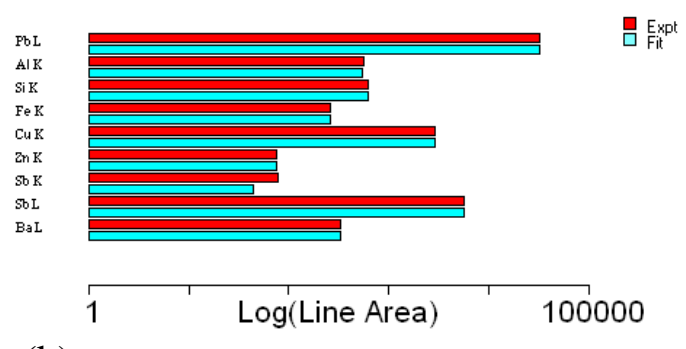

(b)

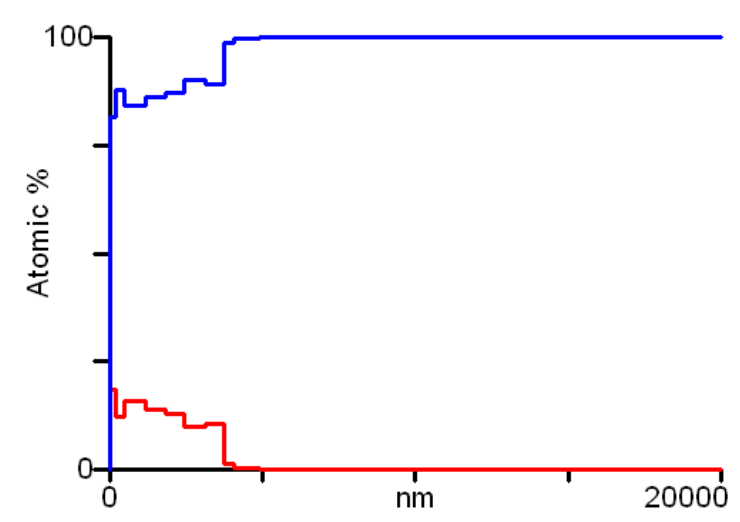

$\mathrm{Pb} 76 \mathrm{Al} ?=5$

C $801 \mathrm{~Pb}$

(c)

Figure 3 : (a) fitted RBS spectrum (b) simultaneously fitted PIXE spectrum and (c) corresponding depth profile for a particle of a smaller diameter than the beam

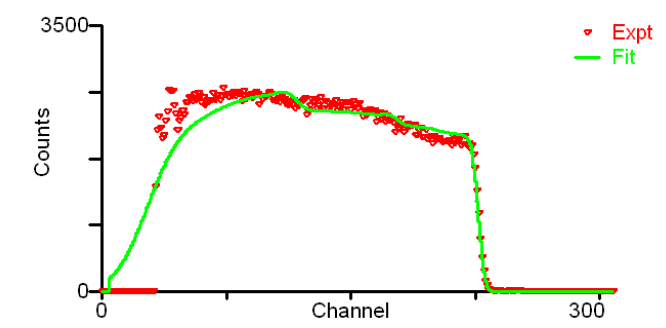

(a)

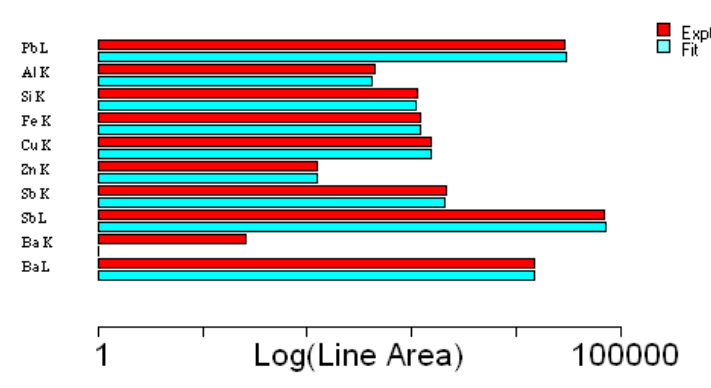

(b)

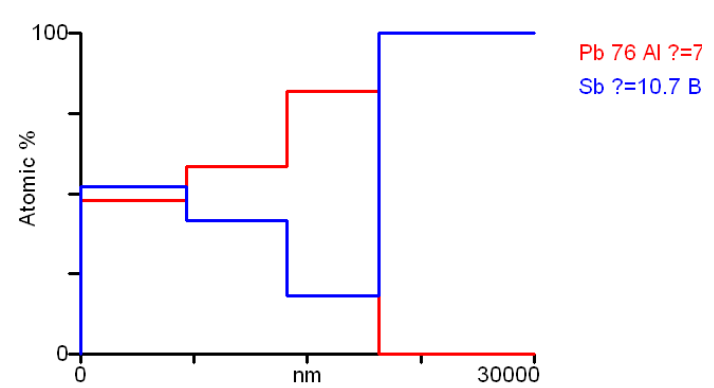

(c)

Figure 2 : (a) fitted RBS spectrum (b) simultaneously fitted PIXE spectrum and (c) corresponding depth profile for a particle of a larger diameter than the beam 


\section{References}

[1] F S Romolo et al, Forensic Sci. Int, 119 (2001) 195-211

[2] Z Brozek-Mucha et al, Forensic Sci. Int. (2001), p.39-47

[3] Reis MA, Alves LC, Jesus AP, Matrix effects correction for quantitative TTPIXE analysis, Nucl. Instrum. Methods Phys. Res., Sect. B, 1996; 109/110: 134-138

[4] Pascual-Izarra C, Reis MA, Barradas NP, Simultaneous PIXE and RBS data analysis using Bayesian inference with the DataFurnace code, Nucl. Instrum. Methods Phys. Res., Sect. B, 2006; 249: 780-783

[5] Jeynes C, Barradas NP, Marriott PK, Boudreault G, Jenkin M, Wendler E, Webb RP, Elemental thin film depth profiles by ion beam analysis using simulated annealing - a new tool, J. Phys. D: Appl. Phys. 2003; 36: R97-R126

[6] Carlos Pascual-Izarra, Nuno P. Barradas, Miguel A. Reis, Chris Jeynes, Michel Menu, Bertrand Lavedrine, Jean Jacques Ezrati, Stefan Röhrs, Towards truly simultaneous PIXE and RBS analysis of layered objects in cultural heritage. Nucl. Instrum. Methods Phys. Res., Sect. B, 2007; 261: 426-429

[7] Beck L, Jeynes C, Barradas NP, Characterization of paint layers by simultaneous selfconsistent fitting of RBS/PIXE spectra using simulated annealing, Nucl. Instrum. Methods Phys. Res., Sect. B, 2008; 266: 1871-1874

[8] J.C.G. Jeynes, C. Jeynes, K.J. Kirkby, M. Rümmeli, S.R.P. Silva, RBS/EBS/PIXE measurement of single-walled carbon nanotube modification by nitric acid purification treatment, Nucl. Instrum. Methods Phys. Res., Sect. B, 2008; 266: 1569-1573

[9] M Webb and C Jeynes, presented at ICMA 2008, Submitted to NIMB

[10] Private communication with C. Moynehan, LGC Forensics

[11] M Webb, D. Cox, K. J. Kirkby and C. Jeynes, Trace Element Profiling of Gunshot Residues by SEM-EDS and PIXE: a Comparative Study, presented at EXRS2008, submitted to J. X Ray Spectrometry

[12] Romolo, F., Margot, P., Forensic Sci. Int. 2001; 119: 195-211

[13] A. Simon, C. Jeynes, R.P. Webb, R. Finnis, Z. Tabatabian, P.J. Sellin, M.B.H. Breese, D.F. Fellows, R. van den Broek and R.M. Gwilliam, Nucl. Instr. and Meth. B, 2004; 219: 405-409

[14] G.W.Grime, Nucl. Instrum. and Meth B, 1996, 109: 170

[15] Bruno Cardinetti, Claudio Ciampini, Carlo D’Onofrio, Giovanni Orlando, Luciano Gravina, Francesco Ferrari, Donatello Di Tullio and Luca Torresi, X-ray mapping technique: a preliminary study in discriminating gunshot residue particles from aggregates of environmental occupational origin, Foren. Sci. Int. 1431 pp.1-19 2004 doi:10.1016/j.forsciint.2004.01.019 